\title{
EXPLORATION OF RADIATING AEROSTRUCTURES ULTIMATE ANTENNA AND STRUCTURE INTEGRATION - EMUS 2019
}

\author{
YURI E. J. KONTER ${ }^{\dagger}$ ，CHARLY HEUTS* AND CEES VAN HENGEL ${ }^{n}$ \\ ${ }^{\dagger}$ Netherlands Aerospace Centre (NLR) \\ Voorsterweg 31, 8316 PR Marknesse, Netherlands \\ * Fokker Elmo \\ Aviolandalaan 33, $4631 \mathrm{RP}$, Netherlands \\ ${ }^{n}$ Fokker Aerostructures \\ Industrieweg 4, 3351 LB Papendrecht, Netherlands
}

Key words: GNSS, VHF, FML, Integration, GLARE

\begin{abstract}
Two antenna designs are described that are both fully integrated into Fiber Metal Laminate fuselage panels. Full integration in this context means that the antennas are flush with the fuselage skin on the outside of the aero structure, while maintaining the structural properties of the FML panel. The antennas are designed to resonate at GNSS and at VHF frequency bands. Design considerations are discussed and preliminary results are presented.
\end{abstract}

\section{INTRODUCTION}

Currently antennas are mounted as separate components onto the exterior of fuselages. These antennas protrude into the airflow around an aircraft which produces drag during flight. Integration of the antennas into the fuselage will further smoothen the outside of aero planes and thus reduce the aerodynamic drag.

Fiber Metal Laminate (FML) is a composite aircraft construction material, consisting of metal layers bound together by glass fiber layers and resin to form a cohesive laminate. Since the glass fiber layers are electrically insulating, applying RF currents onto the metal layers will cause electric fields which can be used to create antennas.

This paper discusses integration of a global navigation satellite system (GNSS) antenna and a VHF antenna into FML panels. The antennas are realized as a double patch antenna and a slot antenna respectively. Stacked patch antennas share many similarities with FML panels in terms of buildup and the electric properties of the used materials. Consequently, an antenna can be made directly in the FML panel with slight modifications to the buildup of the FML layers. Integration of the GNSS antenna is described in section two. Alternatively, a hole can be made in a metal layer which will operate as a slot antenna. A slot can be used to create a VHF antenna. The integration of such a slot antenna into a FML panel is described in section three. 


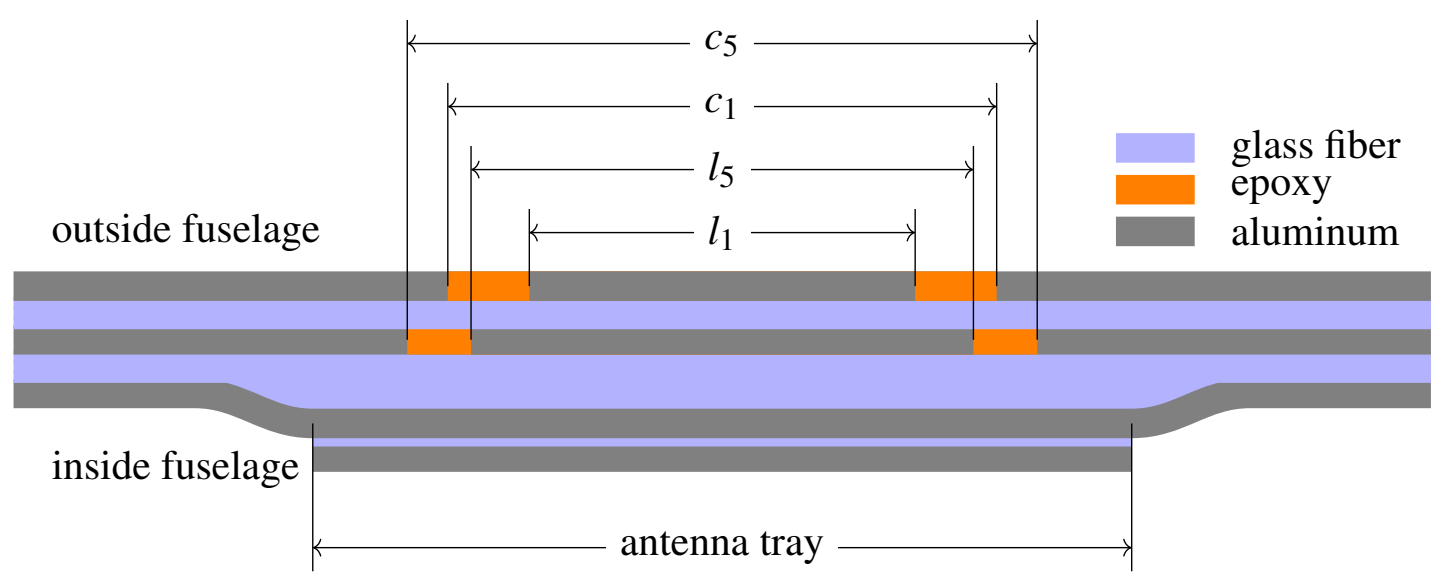

Figure 1: Cross section of the circular double patch GNSS antenna integrated into a FML pane1. The inner aluminum layer of the FML panel is bend to create a tray for the antenna. The two radiating patches are created in-plane with the two outer aluminum layers.

\section{Integration of a double stack GNSS antenna into FML}

GNSS antennas used for aviation are Right Handed Circular Polarized (RHCP) and operate in both the $\mathrm{L} 1$ and the 5 bands. Therefore, a double stacked patch antenna is designed such
that each of the stacked patches radiates in one of the bands. As disctisse in [1], four probes
are used in order to meet the polarization requirements of the GNSS antenna. By doing so,
a good axial ratio is guaranted for a broad viewing angle, whilst keeping the antenna design
robust enough for integration in FML panels.

The antenna is integrated in GLARE G-4B-3/2-0.3 fuselage, a composite material build up Register from three aluminum layers bound together with layers gf olass fiber and resin A circular inner and middle layers. This inner metal layer will function as an electrical ground-plane for the stacked patch antenna. Concentric circular patches are created in the middle and outer aluminum layers resulting in the FML stack shown in figure 1 . The $l_{1}$ and $l_{5}$ patches will attribute to radiation in the L1 and L5 bands respectively. An additional aluminum layer is added on the inside of the dent to provide additional strength to the fuselage. This aluminum layer has no effect on the electrical performance of the integrated antenna.

\subsection{Fabrication tolerances}

The GNSS antenna is made by placing the circular patches in an autoclave together with the fuselage panels. In doing so, the antenna becomes a flush integral part of a fuselage panel. During this manufacturing process, the metal components responsible for the antenna function of the fuselage panel can drift. Two fabrication artifacts are of particular interest. The centers of the circular patches can be misaligned (figure $2 \mathrm{a}$ ) and fuselage can drift towards the antenna patches (figure $2 b$ ). The effect of these deviations are shown in figure 3. The FML panels can drift several millimeters with no significant consequence on the antenna performance. Drifts of the FML panels relative to the circular antenna patches primarily affect antenna performance 


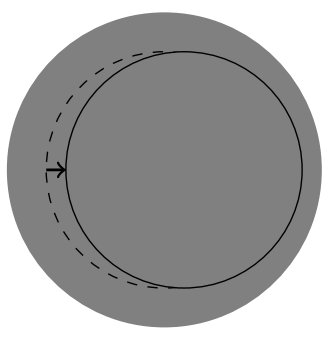

(a) The two circular metal patches can show some misalignment resulting in degradation of antenna performance.

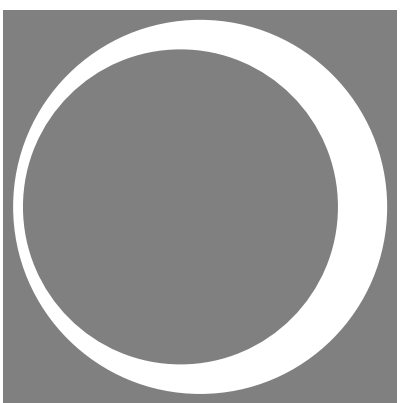

(b) The circular antenna patches can drift towards the fuselage panel which can lead to unwanted electrical coupling between the patch and the fuselage panel.

\section{Figure 2: Examples of misalignment resulting from the fabrication process.}
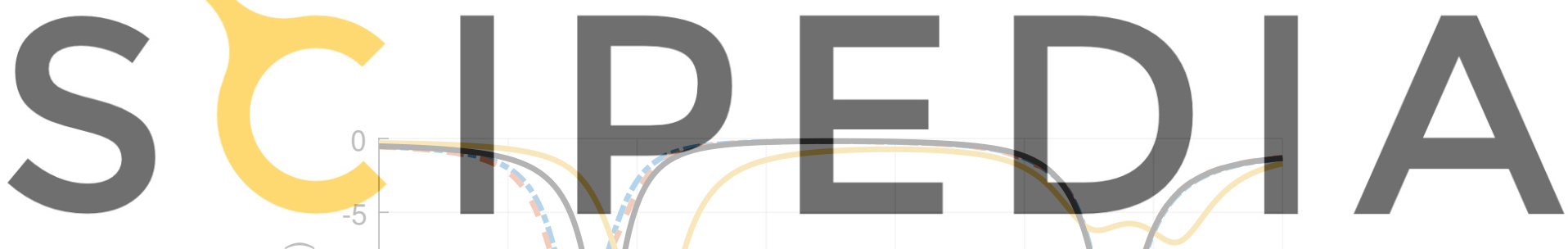

Register for free 能 hotps//www.scipedia.com to download the version without the watermark

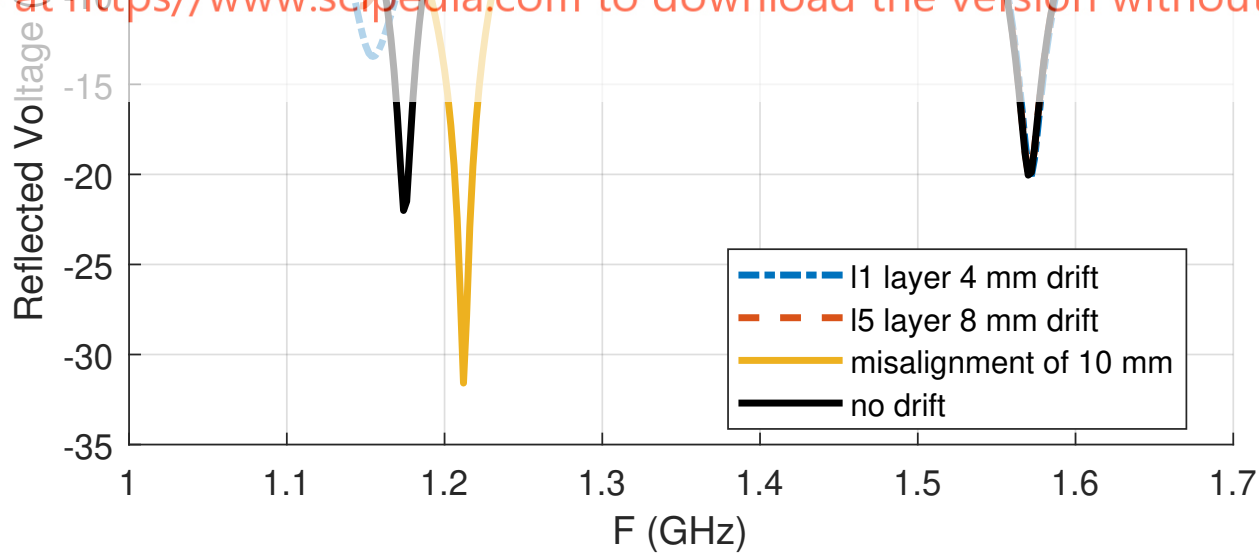

Figure 3: The effect of misalignment on the reflected voltage of the integrated antenna. The two resonance peaks correspond to the L5 band (left) and the L1 band (right). The reflected voltage subject to drifts of the middle FML metal layer and outer FML metal layer are shown in blue and red respectively. The effect of misalignment of the circular patches is shown in yellow. 


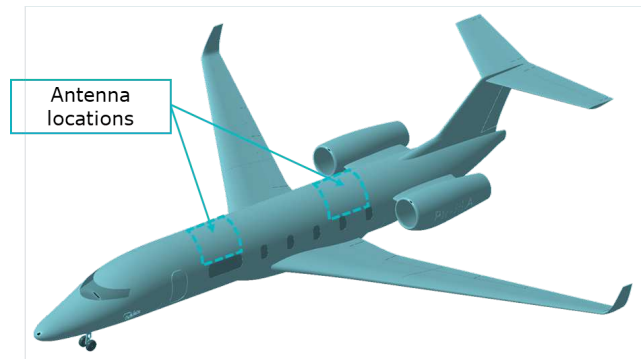

(a) Location of VHF slot antennas on outside of aircraft.

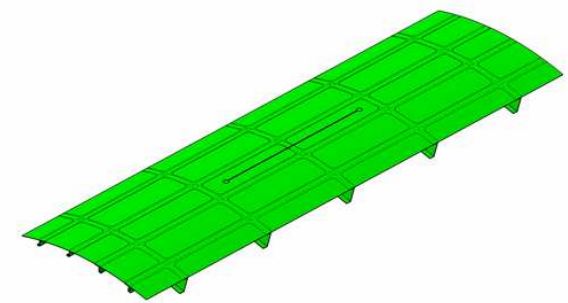

(b) VHF slot antenna integrated between frames and stringers of GLARE fuselage panel.

Figure 4: Integration of VHF slot antenna.

in the L5 band. The loss in performance due to drifts of the middle FML metal layer is due to coupling between the L5 radiating patch and the fuselage. The performance loss caused by drifts of the outer metal layer is caused by the outer FML metal layer which partially covers the lower circular patch. Misalignment of the two antenna patches increases the parasitic coupling between the two patches, improving the performance of the inner patch. However, the resonant when the L1 circular p patch.

The observed detrimental are large enough. These threshold
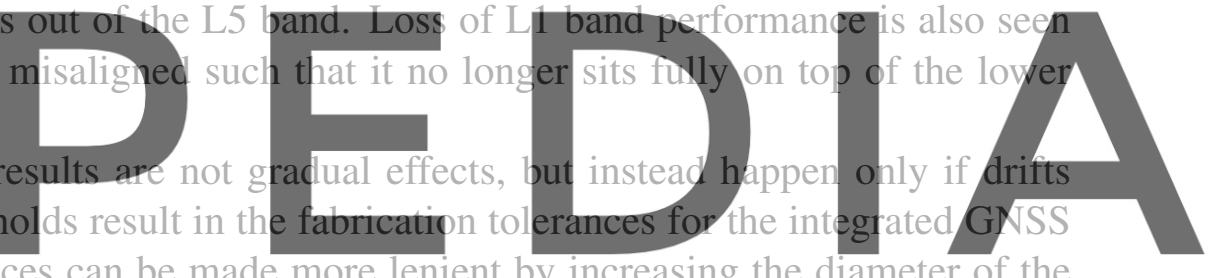

antenna. The imposed tolerances can be made more lenient by increasing the diameter of the

Register for free at hteps//www.scipedia.com to download the version without the watermark

\section{Integration of a VHF slot antenna into FML}

The larger wavelength, and therefore larger antenna sizes, make that VHF antennas are of particular interest for miniaturization. A slot antenna allows flush integration of antennas into the fuselage regardless of the operating frequency and wavelength. Moreover, FML has a large metal area on the outside of the fuselage which is required for proper operation of a slot antenna.

Slot antennas radiate in both directions away from the conducting surface, resulting in unwanted radiation into the inside of the aircraft. The inward radiation can be suppressed by introducing a ground plane shield a quarter wavelength $(75 \mathrm{~cm})$ behind the antenna. This required length can be reduced significantly by applying a parallel plate resonator as radiation shield [2]. Additionally, the bandwidth of a slot antenna is improved by applying a feed technique where two separate modes of the slot are excited [3].

\subsection{Integration of the VHF antenna}

Vertical polarization is achieved with a horizontally oriented slot antenna placed on the sides of an aircraft as shown in figure 4a. Due to the length of the slot needed for VHF radiation, the slot will run through one of the frames as shown in figure $4 \mathrm{~b}$. Modification to the frames 


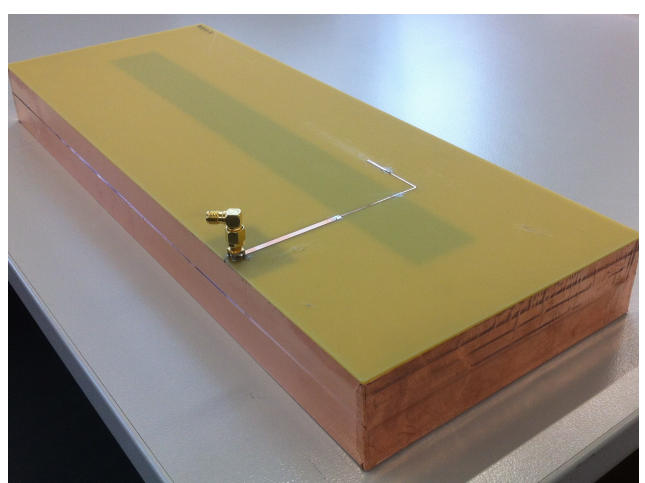

(a) Slot antenna with a folded parallel plate resonator. The antenna is made using standard FR4 as dielectric with copper plating. The rectangular slot is visible as the shaded area in the top FR4 layer.

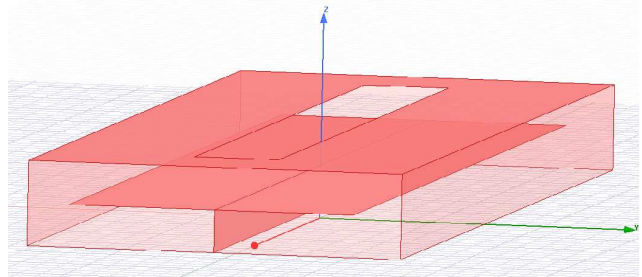

(b) Design showing fold of the PPR. The PPR fold is created by placing a T-shaped copper structure underneath the slot.

\section{Figure 5: $300 \mathrm{MHz}$ slot antenna with PPR to suppress back radiation.}

are required to fit the PPR behind the slot on the inside of the fuselage. These modifications

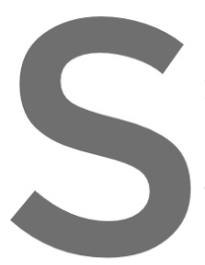

should preserve both

fuselage panel.

3.2 Verification of
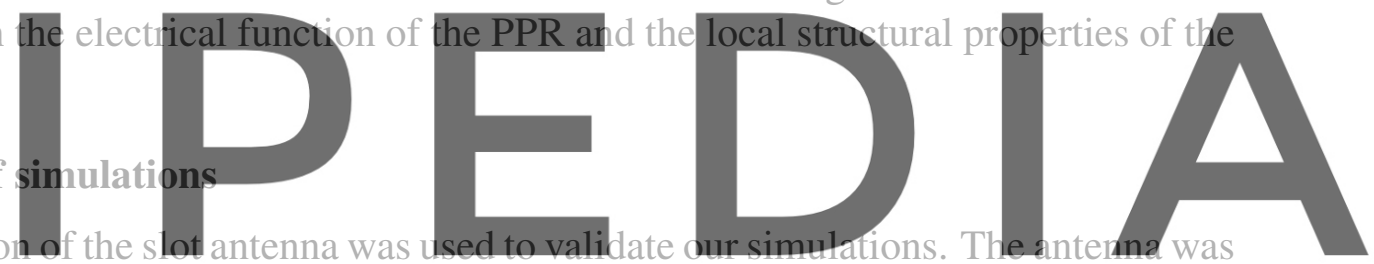

A $300 \mathrm{MHz}$ version of the slot antenna was used to validate dur simulations. The antenna was
de using FR4 materials as shown in figure 5a). The PPR is folded according to figure $5 \mathrm{~b}$. The

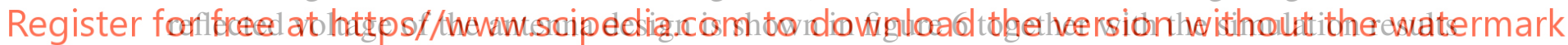
created in FEKO. The two resonant modes required to broaden the bandwidth are recognizable by the valleys in reflected voltages. Increasing the length of the stub reduces the reflected voltage for the lower resonant frequency of about $270 \mathrm{MHz}$ but increases the reflected voltage of the higher resonant frequency at about $340 \mathrm{MHz}$. Thus, a trade-off is made resulting in the broadest possible bandwidth.

\section{Conclusion}

Two fully integrated antenna concepts are presented, both with a flush finish on the outside of the fuselage. Effects of integration on electrical performance have been simulated resulting in fabrication limits for the antenna designs. The performance of the VHF slot antenna with a PPR has been demonstrated with a prototype. Measurements of the prototype show good correspondence with the simulations results. The antennas will further improve aerodynamic properties of aero structures by replacing protruding electrical components with flush integrated antenna solutions. 


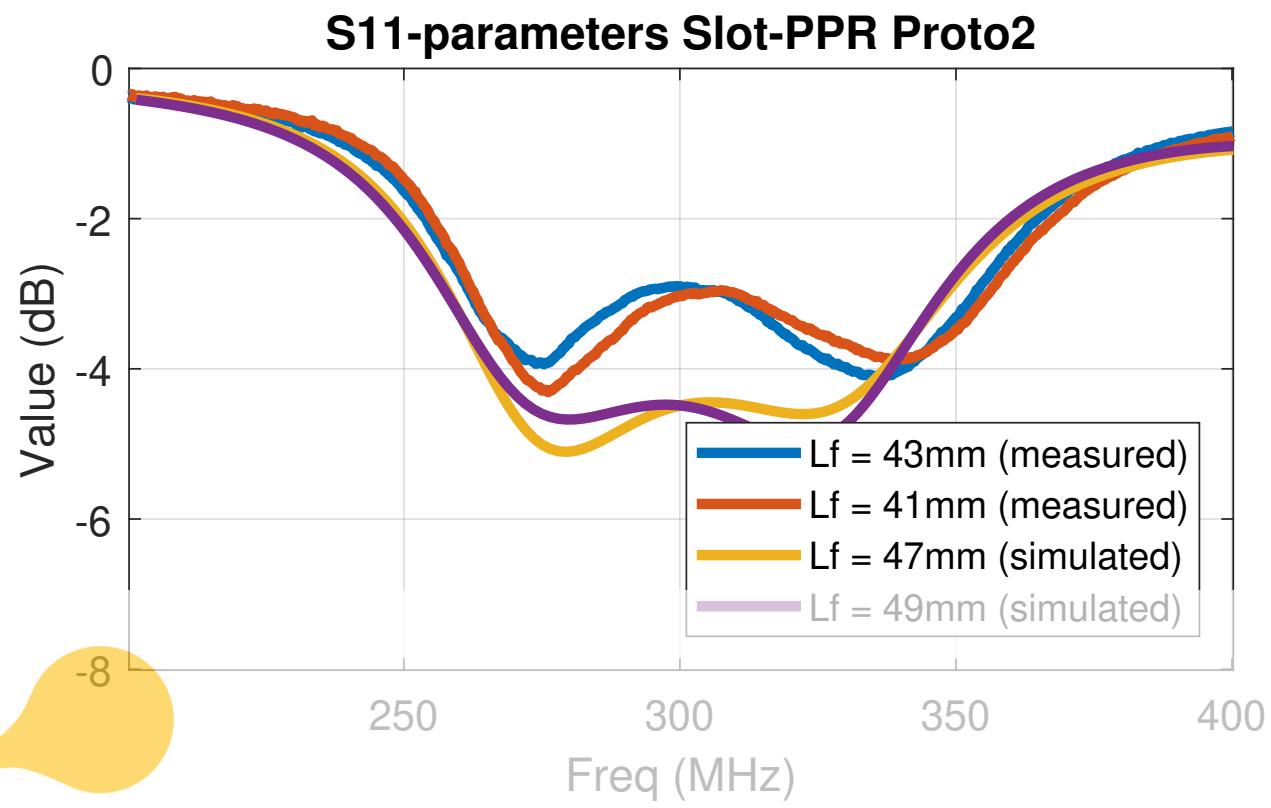

Figure 6: Simulated and measured reflected voltage the $300 \mathrm{MHz}$ slot antenna with a folded parallel plate resonator as shield. The length of the stub is given by $L f$.
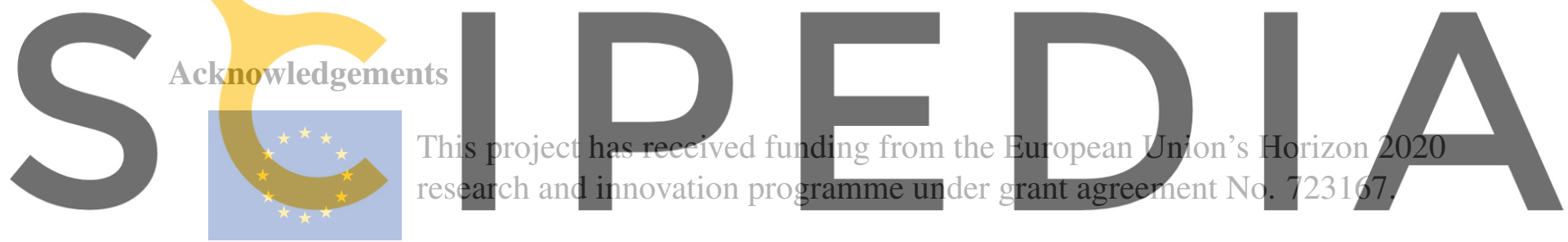

Register for free at https//www.scipedia.com to download the version without the watermark

[1] K. Yinusa, "A Dual-Band Conformal Antenna for GNSS Applications in Small Cylindrical Structures," IEEE Antennas and Wireless Propagation Letters, pp. 1-1, 2018.

[2] W. Hong and K. Sarabandi, "Platform Embedded Slot Antenna Backed by Shielded Parallel Plate Resonator," IEEE Transactions on Antennas and Propagation, vol. 58, pp. 28502857, Sept. 2010.

[3] N. Behdad and K. Sarabandi, "A wide-band slot antenna design employing a fictitious short circuit concept," IEEE Transactions on Antennas and Propagation, vol. 53, pp. 475-482, Jan. 2005. 